\title{
Colorful Pigments for Hair Dying Based on Enzymatic Oxidation of Tyrosine Derivatives
}

Yuhe Shen, ${ }^{\dagger}$ Jiayu Liu, ${ }^{\dagger}$ Yuefei Wang, ${ }^{\dagger, \S}, *$ Wei Qi, ${ }^{\dagger, t, \S, *}$ Rongxin Su, ${ }^{\dagger},+, \S$ Zhimin He $^{\dagger}$

$\dagger$ School of Chemical Engineering and Technology, State Key Laboratory of Chemical Engineering, Tianjin University, Tianjin 300072, P. R. China

${ }^{\ddagger}$ Collaborative Innovation Center of Chemical Science and Engineering (Tianjin),

Tianjin 300072, P. R. China

$\S$ Tianjin Key Laboratory of Membrane Science and Desalination Technology, Tianjin University, Tianjin 300072, P. R. China.

*Corresponding author: Yuefei Wang (email: wangyuefei@tju.edu.cn) and Wei Qi (email: qiwei@tju.edu.cn). 


\section{Supporting Figures}

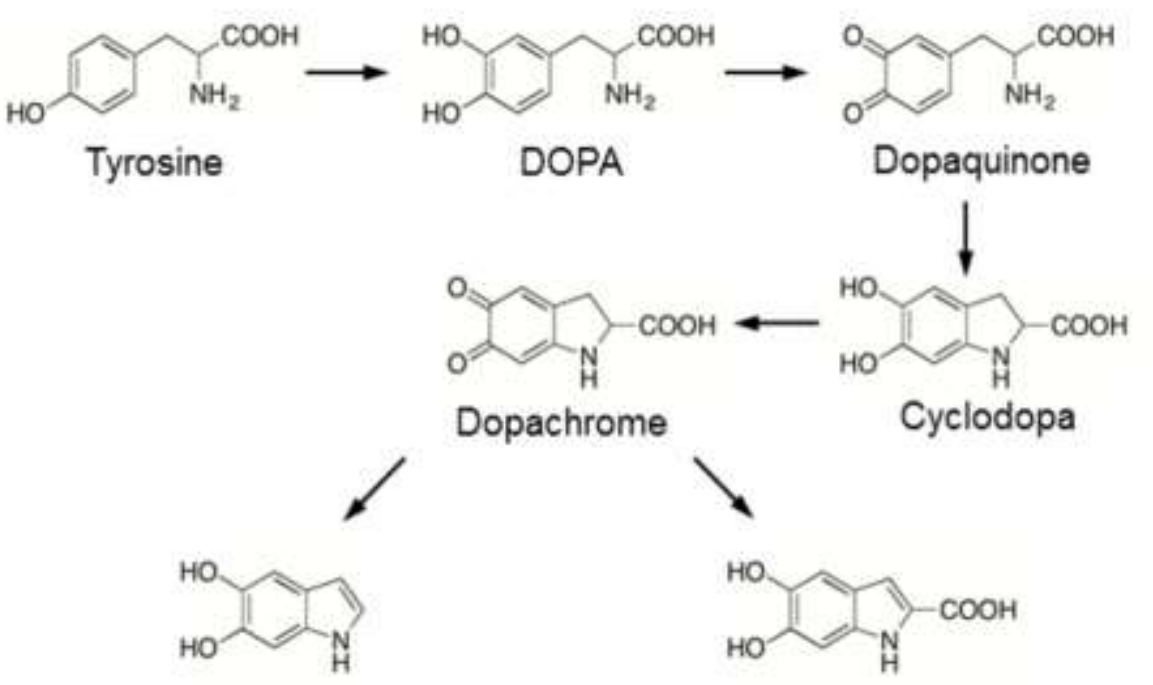
5,6-Dihydroxyindole $\quad 5,6$-Dihydroxyindole-2-carboxylic acid (DHI) (DHIC)

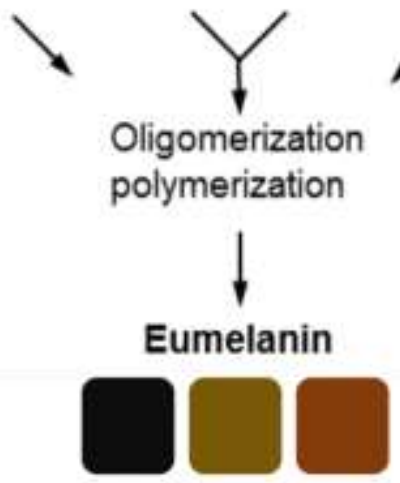

Figure S1. Mechanism of chemoenzymatic oxidation of tyrosine ( ${ }_{L}-\mathrm{Tyr}$ ) to eumelanin occurring inside the melanocytes. 


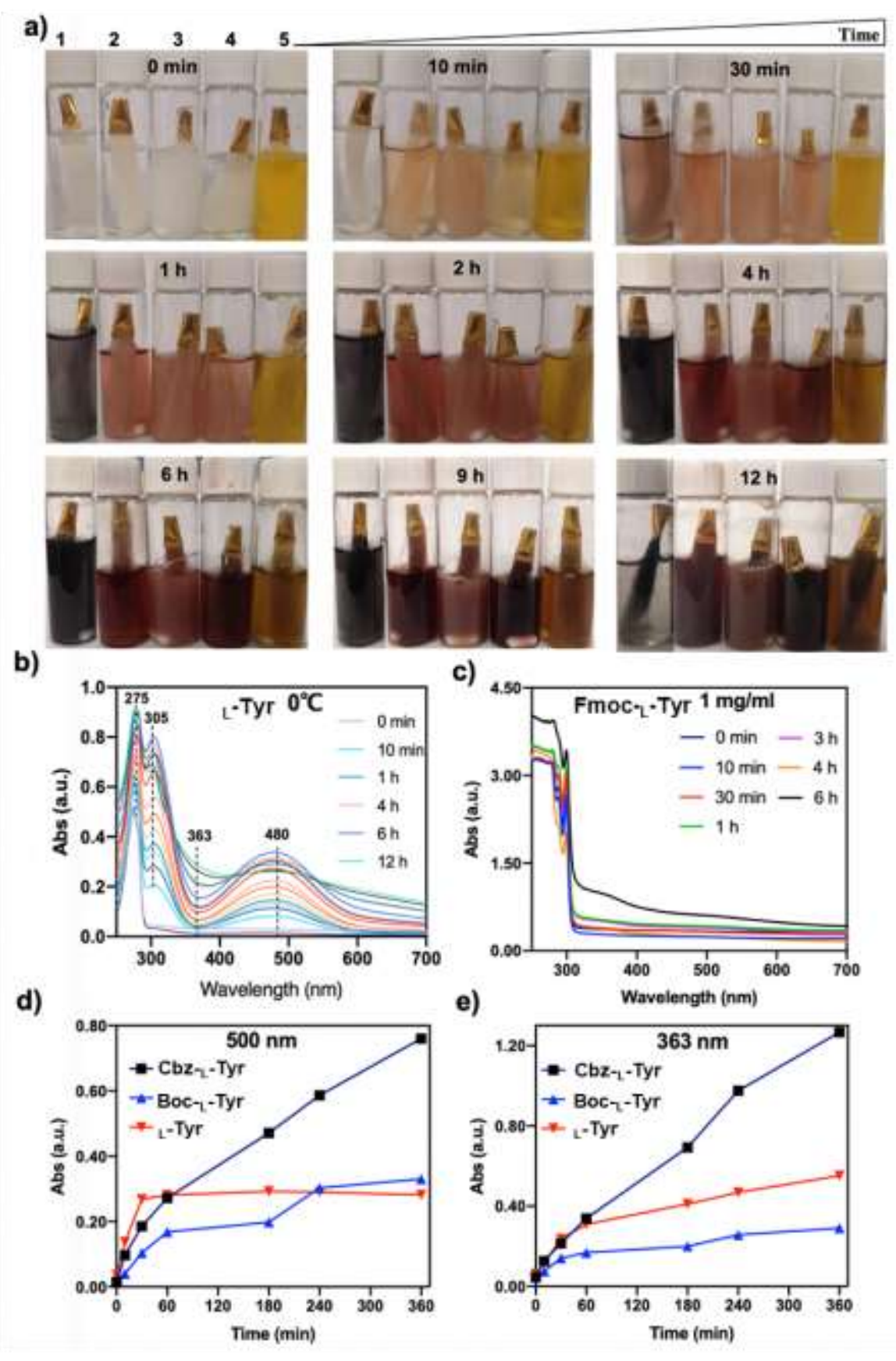

Figure S2. a) The rich colors and their corresponding UV spectrum produced by the oxidation at $37^{\circ} \mathrm{C}$ of different tyrosine and derivative molecules $1-5$, from left to right are L-Tyr, Boc-L-Tyr, Fmoc-L-Tyr, Cbz-L-Tyr and Fc-L-Tyr. Over time, coloring is achieved on the surface of the hair; b) The UV spectrum of the slowed-down oxidation reaction of tyrosine at $0^{\circ} \mathrm{C}$, and the chromophore has three $\lambda \max$ at $275 \mathrm{~nm}, 305 \mathrm{~nm}$ and $480 \mathrm{~nm}$; c) UV-Vis spectra of the supernatant of Fmoc-L-Tyr during enzymatic oxidation at $37^{\circ} \mathrm{C}$; d-e) the reaction kinetic curves of three molecules at 363 and $500 \mathrm{~nm}$, which represent the intermediate red substance and Eumelanin respectively to further 
explore the kinetics of tyrosine enzymatic oxidation at $37^{\circ} \mathrm{C}$. The oxidation process of unmodified ${ }_{\mathrm{L}}$-Tyr is the fastest, for it's the first to produce intermediate red substance absorption peak, and after a short peak change the entire spectrum will be absorbed. As time goes by, the $500 \mathrm{~nm}$ absorption peak gradually tends to decrease and the Eumelanin absorption peak at $363 \mathrm{~nm}$ is continuously strengthened and stabilized. On the contrary, the enzymatic oxidation of Boc- $-\mathrm{L}-\mathrm{Tyr}$ is slower, its product peak remains at $500 \mathrm{~nm}$ and will not disappear with time, therefore the red substance produced could be retained for a longer time. While the peak changes of Cbz-L-Tyr and other derivatives are obvious, and the characteristic peaks at 363 and $500 \mathrm{~nm}$ are constantly increasing, leading to the product presents a mixed red-orange color. This initially verifies our conjecture that the oxidation state of tyrosine will produce different color transitions and the obvious deepening of the solution during the reaction indicates the successful polymerization of the amino acid monomer. 

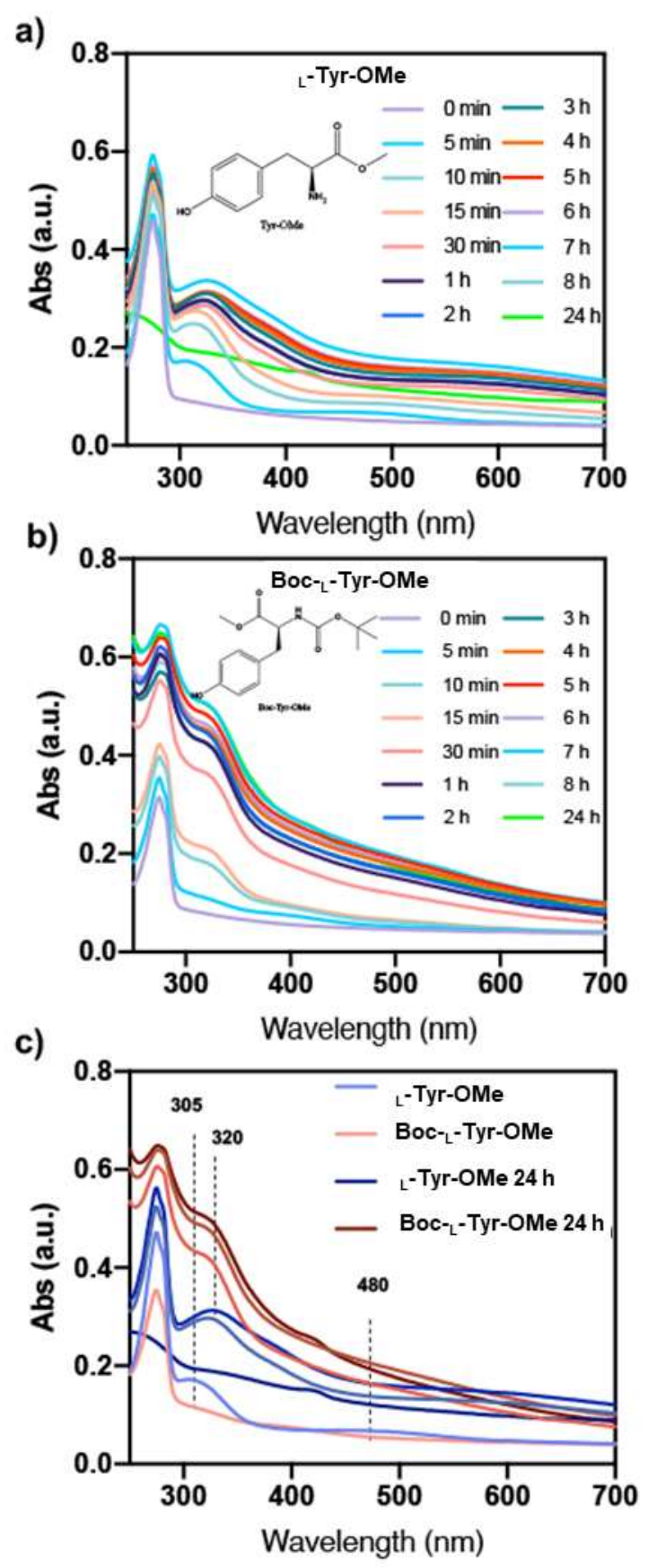

Figure S3. a, b) UV-Vis spectrum of the supernatant of ${ }_{\mathrm{L}}-\mathrm{Tyr}-\mathrm{OMe}$ and Boc-L-Tyr-OMe chemoenzymatic oxidation reactions carried out at $37^{\circ} \mathrm{C}$ and c) the comparison diagram

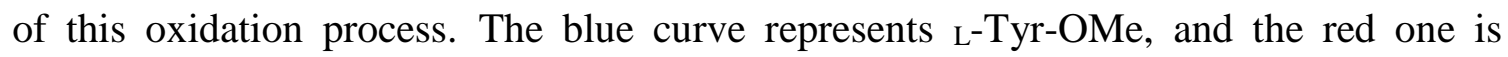
Boc-L-Tyr-OMe. The color of the curve from light to dark represents the progress of the oxidation reaction. There are obvious differences in the oxidation rate and characteristic peaks of the two, and the reaction is complete. Later, the UV absorption of the two is completely different (the darkest curve). 

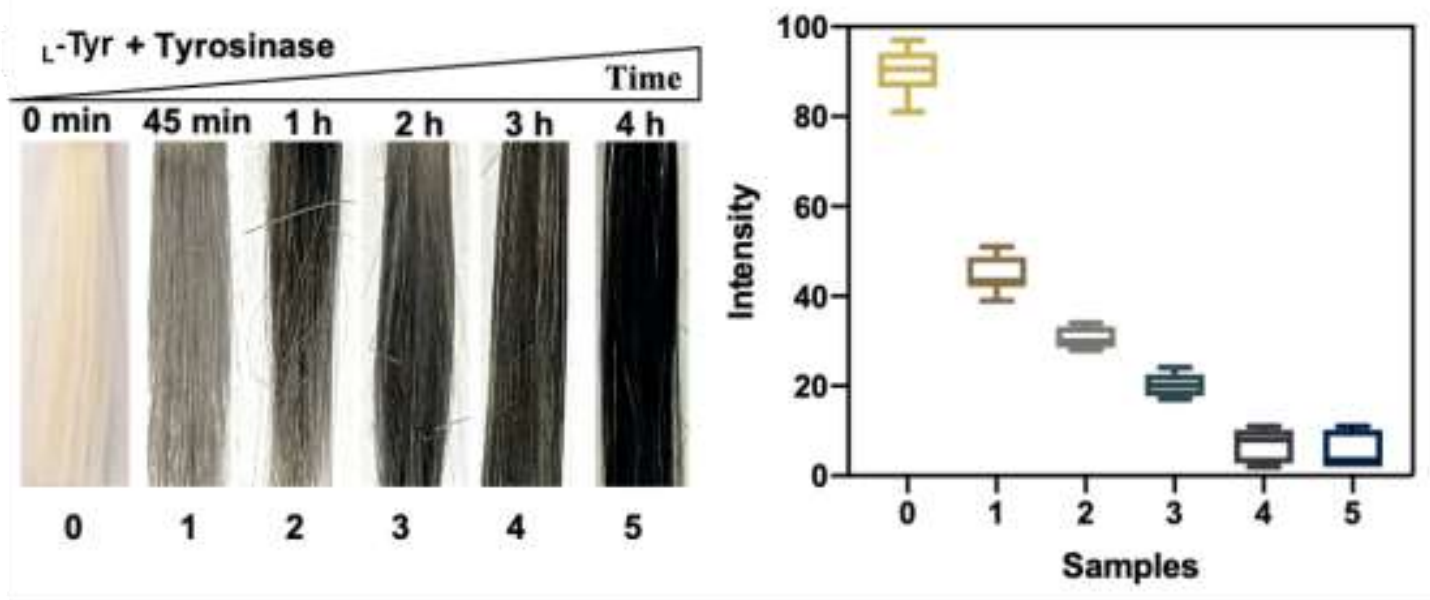

Figure S4. Diagram of the effect of tyrosine oxidation time on hair dyeing effect and image analysis (color intensity) of photographs of hair 1-5.
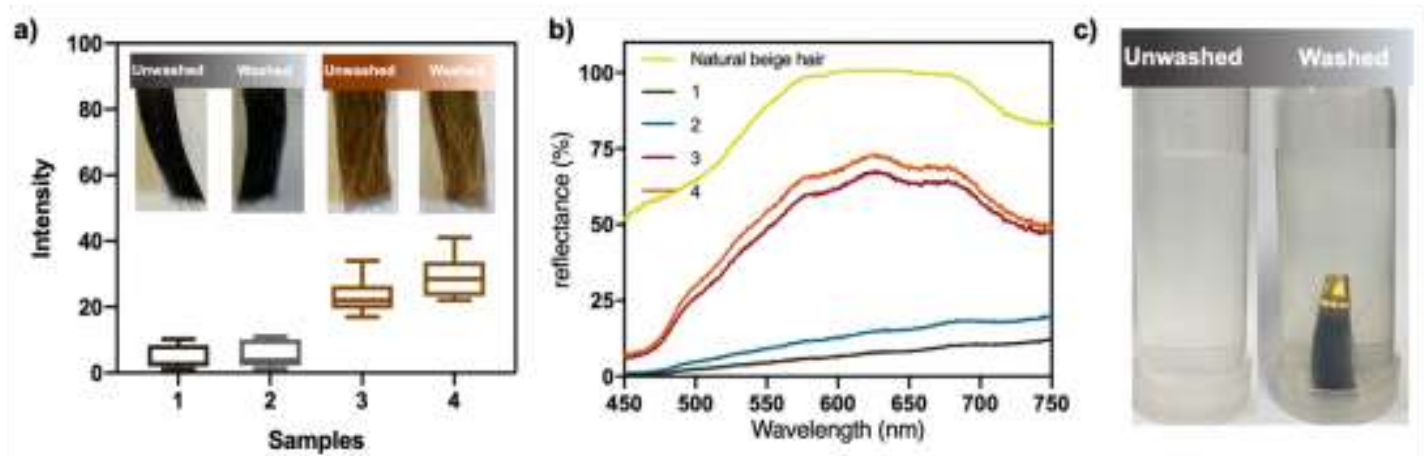

Figure S5. Retention of hair color after 20 washes with shampoo. a) Photographs of hair dyed with ${ }_{\mathrm{L}}-\mathrm{Tyr}$ and Boc-L-Tyr using tyrosinase at $37^{\circ} \mathrm{C}$ before and after 20 washes with a $10 \%$ shampoo solution, and their color intensity analysis. b) Color reflectance analysis of hair before and after washes. c) Comparison between the color of the soiled solution from synthetic melanin dyed hair before and after washing. 


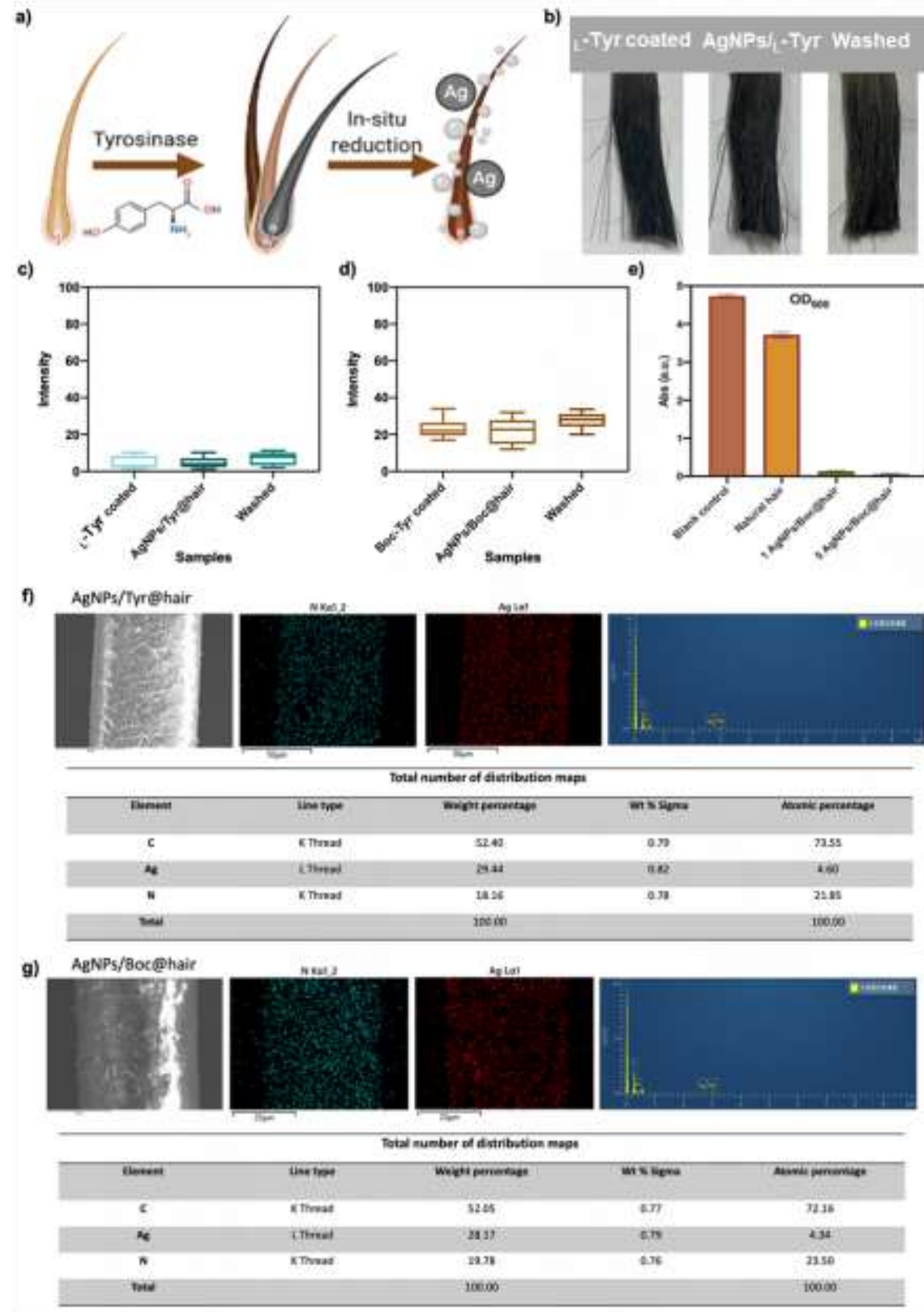

Figure S6. a) Schematic diagrams of AgNPs/ Tyr@hair synthesis procedures. b) Macro photo of hair after L-Tyr coating, and then further adhesion of silver nanoparticles and washing. c, d) The color intensity analysis of the surface of ${ }_{\mathrm{L}}-\mathrm{Tyr}$ and Boc-L-Tyr during this whole experiment. e) The $\mathrm{OD}_{600}$ value change curve of agar plates characteristic of the antimicrobial activities of AgNPs/ Boc@hair against E. coli. f, g) SEM image and EDS analysis of the typical f) AgNPs/ Tyr@hair and g) AgNPs/ Boc@hair, there is no significant difference in the amount of silver loaded between them. 
Table S1. Macro photos of solutions of various tyrosine derivatives after enzymatic oxidation in different states and their parameter analysis by "Lab color model".

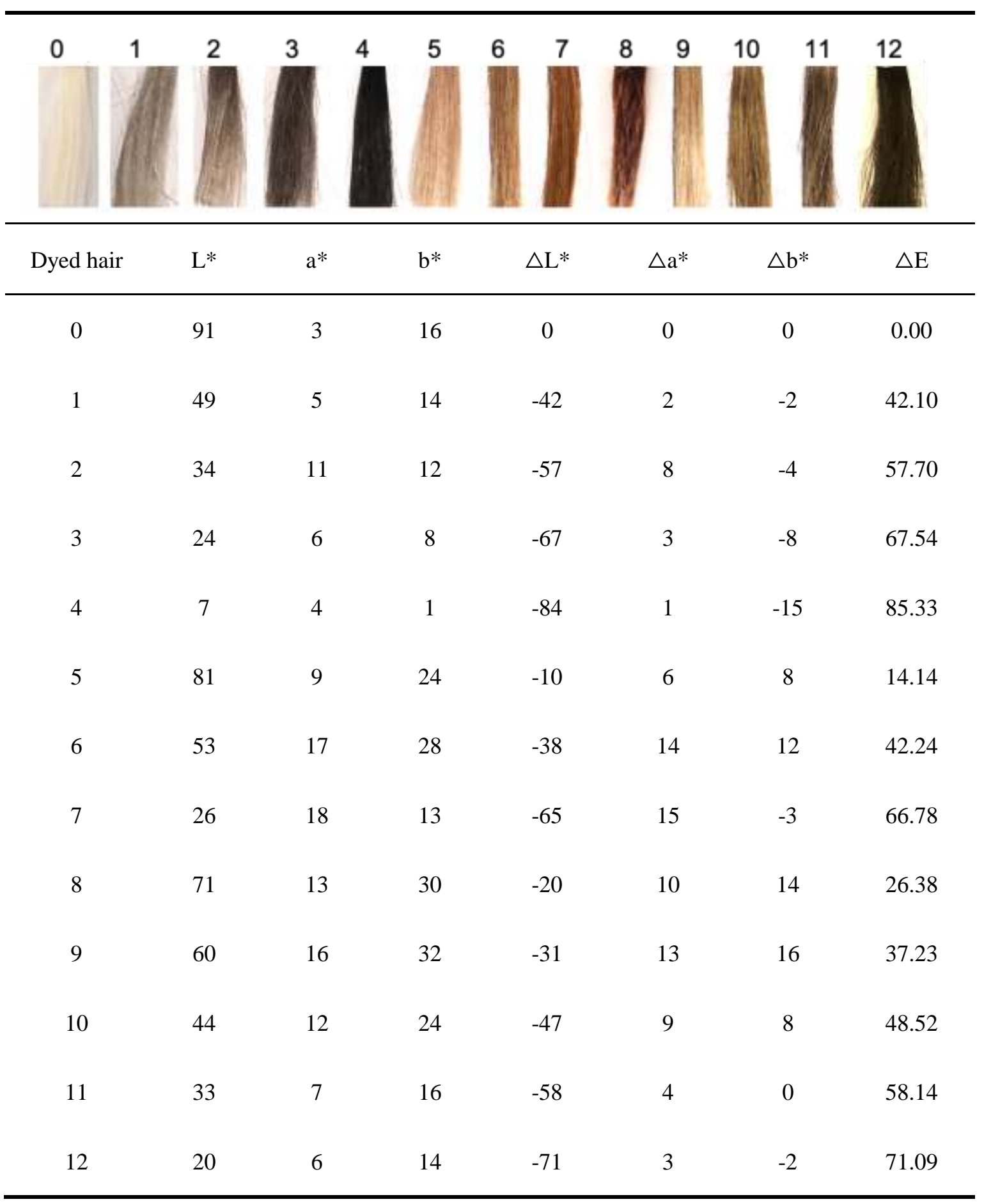

